\title{
Ferroelastic Phase Transition in a Layered Bismuth Oxychloride Single Crystals
}

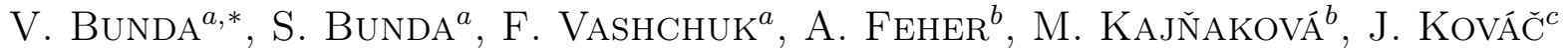 \\ AND E. SYRKIN ${ }^{d}$ \\ ${ }^{a}$ Faculty of Information Sciences, Transcarpathian State University, Zankovetska St. 89-A, Uzhgorod 88 015, Ukraine \\ ${ }^{b}$ Center of Low Temperature Physics, Faculty of Science, P.J. Šafarik University \\ Park Angelinum 9, 04154 Kosice, Slovakia \\ ${ }^{c}$ Institute of Experimental Physics, Slovak Academy of Sciences, Watsonova 47, Kosice, Slovakia \\ ${ }^{d}$ Verkin Institute for Low Temperature Physics and Engineering of the National Academy of Sciences of Ukraine \\ 47 Lenin Ave., Kharkov, Ukraine
}

\begin{abstract}
We report the results of low-temperature specific-heat, magnetization, and X-ray diffraction measurements on a bismuth oxyhalide $(\mathrm{BiOCl})$ single crystal. We conclude that $\mathrm{BiOCl}$ in temperature region $150-230 \mathrm{~K}$ shows "antiferroelastic-paraelectric" phase transitions at critical temperatures $T_{\mathrm{c} 1}=167.5 \mathrm{~K}$ and $T_{\mathrm{c} 2}=214.6 \mathrm{~K}$, respectively.
\end{abstract}

PACS numbers: 64.70.K-, 81.30.-t, 65.40.-b

\section{Introduction}

Bismuth oxychloride $\mathrm{BiOCl}$ single crystals are very interesting materials which find various applications in photonics. The great interest for these materials is strongly related to the influence of dimensionality on the behaviour of physical properties, polymorphic and phase transformations [1].

\section{Experimental details}

The $\mathrm{BiOCl}$ compound was prepared by dissolving 99.99 pure bismuth oxides in hydrochloric acid, evaporating to form the hydrated chlorides, dehydrating the latter under the vacuum, heating at $\approx 380-700^{\circ} \mathrm{C}$ in a $\mathrm{Cl}_{2}$ atmosphere, cooling to room temperature, and washing the product to remove $\mathrm{BiCl}_{3}$. The products was $\mathrm{BiOCl}$. The $\mathrm{BiOCl}$ single crystal was grown up using the vapour gas transport reaction method in closed volume. The lattice parameters are $a=3.887 \AA$ and $c=7.354 \AA$. A Laue photograph taken parallel to the large face of the platelets showed clearly a fourfold symmetry axis characterizing (001) planes of a matlockite tetragonal PbFCl-like structure. An examination of the platelets using a polarizing microscope shows no extinction, thus indicating that the tetragonal $c$-axis is perpendicular to the plane of the platelets [1]. The specific heat was measured

\footnotetext{
* corresponding author; e-mail: viktor.bunda@upjs.sk
}

in the temperature range from 2 to $50 \mathrm{~K}$ in zero and $8 \mathrm{~T}$ magnetic field. The measurements were performed in a Physical Properties Measurement System (PPMS) from Quantum Design.

\section{Results and discussion}

$\mathrm{BiOCl}$ structure consists of $\mathrm{O}-\mathrm{Bi}-\mathrm{Cl}-\mathrm{Cl}-\mathrm{Bi}-\mathrm{O}$ layers and belongs to space group of $P 4 / \mathrm{nmm}$. The structure exhibits a cleavage plane perpendicular to the $c$ axis where the bonding between the neighboring $\mathrm{Cl}$ layers is weaker [1]. On the basis of the structure we concluded that the origin of the dipole moment was due to the induced atomic dipole of the halogen, aligned antiparallel with the $c$ axis [2].

For the analysis of specific heat we have chosen the approximation described in detail in the paper of Martin [3]. In this approximation the phonon spectrum is again divided into the acoustic and optical branches and those are described by the modified Debye and Einstein formulae, respectively. The resulting isobaric phonon specific heat can be then written in the form

$$
\begin{gathered}
\left(C_{p}\right)_{\text {calc }}=R\left(\sum_{i=1}^{n} \frac{1}{1-\alpha_{E_{i}} T} \frac{x_{E_{i}}^{2} \exp \left(x_{E_{i}}\right)}{\left[\exp \left(x_{E_{i}}-1\right)\right]^{2}}\right. \\
\left.+\frac{n-3}{1-\alpha_{\mathrm{D}} T}\left(\frac{1}{x_{\mathrm{D}}}\right)^{3} \int_{0}^{x_{\mathrm{D}}} \frac{x^{4} \exp (x)}{[\exp (x)-1]^{2}} \mathrm{~d} x\right),
\end{gathered}
$$

where $\alpha_{\mathrm{D}}$ and $\alpha_{E_{i}}$ are the anharmonic correction coefficients for acoustic and optical branches, respectively; $n$ 


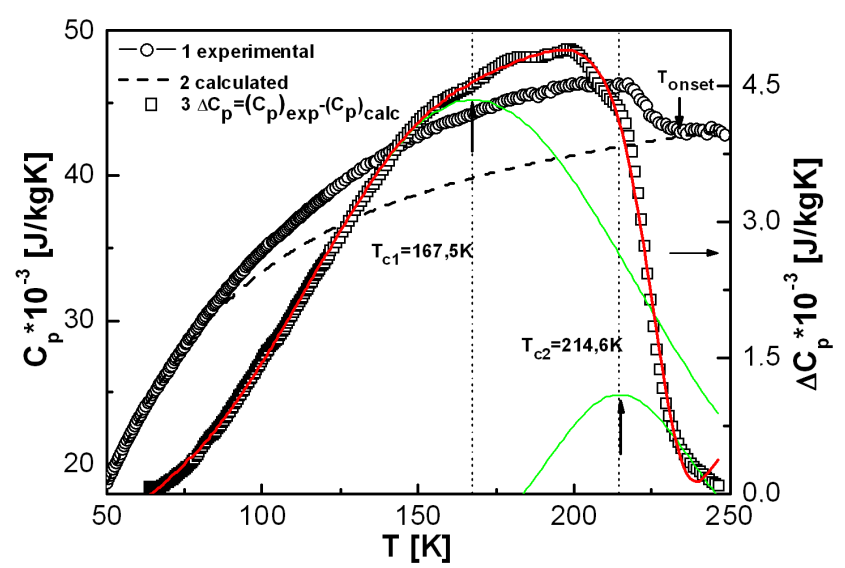

Fig. 1. Temperature dependence of experimental (curve 1), calculated (curve 2) specific heat and their difference (curve 3 ) in region of phase transitions $\mathrm{BiOCl}$ single crystal. Green curves indicate the temperature areas of existence of the ferroelastic domains.

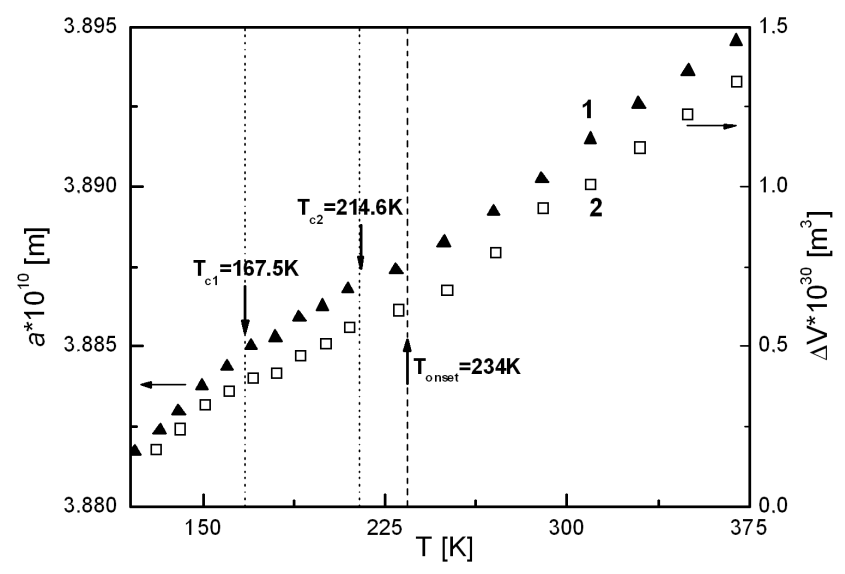

Fig. 2. The temperature dependence of lattice parameter $a$ (curve 1) and change of elementary cell volume of unit cell $\Delta V$ (curve 2) in phase transitions region of $\mathrm{BiOCl}$.
- number of acoustic and optical branches $(n=18$ for $\mathrm{BiOCl}) ; R$ is the gas constant. The temperature dependence of the experimental $\left(C_{p}\right)_{\exp }$ and calculated $\left(C_{p}\right)_{\text {calc }}$ specific heat of $\mathrm{BiOCl}$ in both $C$ vs. $T$ is present in Fig. 1 . The $\Delta C_{p}$ value is difference between $\left(C_{p}\right)_{\exp }$ and $\left(C_{p}\right)_{\text {calc }}$.

From the dielectric constant, specific heat, magnetic susceptibility and X-ray diffraction studies on $\mathrm{BiOCl}$ as a function of temperature, we report the wide maximum in the dielectric constant and specific heat (Fig. 1) and breaks in the X-ray diffraction in temperature region $150-230 \mathrm{~K}$ (Fig. 2). They testify that "antiferroelastic(AFE)-paraelectric(PE)" phase transitions are present possibly by order-disorder mechanism [4] at critical temperatures $T_{\mathrm{c} 1}=167.5 \mathrm{~K}$ and $T_{\mathrm{c} 2}=214.6 \mathrm{~K}$, respectively. The mechanism of "AFE$\mathrm{PE}$ " phase transition in $\mathrm{BiOCl}$ is caused by possible existence of ferroelastic domains on basis of $\mathrm{Bi}-\mathrm{O}$ coordination polyhedron [2].

The results discussed above reveal that it is highly desirable to study the inelastic neutron scattering on $\mathrm{BiOCl}$ to be able to directly measure the dispersion relation of phonons and domains structure.

\section{Acknowledgments}

This work has been supported, in part, by VEGA under grant No. 1/0159/09 and project No. APVV-0006-07.

\section{References}

[1] V.V. Bunda, S.O. Bunda, in: Physics of Electronic Materials, Vol. 2, 2nd Int. Conf. Proc., Kaluga (Russia) 2005, p. 134 .

[2] M. Sieskind, A. Polian, M. Fischer, F. Decremps, J. Phys. Chem. Solids 59, 75 (1998).

[3] C.A. Martin, J. Phys., Condens. Matter 3, 5967 (1991).

[4] H. Oppermann, M. Schmidt, H. Bruckner, W. Schnelle, E. Gmelin, Z. Anorg. Allg. Chem. 626, 937 (2000). 